\title{
Linearity Characteristics Study of Millimeter-Wave GaN Power Amplifier
}

\author{
Joe X. Qiu, A.M. Darwish", E.A. Viveiros, H.A. Hung, and K. Kingkeo \\ U.S. Army Research Laboratory, Adelphi, MD, 20783, USA
}

\begin{abstract}
In this paper, a digital waveform system for evaluating the linearity characteristics of millimeter-wave GaN HEMT devices and MMICs is described. It is capable of generating and analyzing a wide range of waveforms. The linearity performance of a sample Ka-band one-stage $\mathrm{GaN} / \mathrm{SiC}$ MMIC power amplifier is characterized to demonstrate the capabilities of the system. Performance metrics associated with different waveforms are measured. These include AM/AM and AM/PM for single-tone, intermodulation-product-to-carrier-ratio for two-tone, EVM and ACPR for digitally modulated waveforms. The dependence of these metrics on drain bias current is studied. This digital waveform system and its associated test methodology will help MMIC designers to assess device linearity characteristics to improve MMIC PA design with optimized power, efficiency and linearity.
\end{abstract}

Index Terms - Power amplifier, SSPA, MMIC, millimeterwave, GaN, linearity.

\section{INTRODUCTION}

With the increasing need for high-data-rate satellite communication (SATCOM) links and the overcrowding spectrum at Ku-band and below, millimeter-wave ( $\mathrm{mmW}$ ) frequencies have become an appropriate solution to provide wider bandwidth and higher date throughput for both commercial and defense satellite applications [1][2]. The availability of high-power, high-efficiency, linear amplifiers at these frequencies is important for the successful deployment of these systems. Traditionally, SATCOM applications have relied on traveling-wave-tube amplifiers (TWTAs) to satisfy their transmitter power requirement. During the past decade, the emergence of wide-bandgap semiconductor devices has enabled solid-state power amplifiers (SSPAs) to potentially replace TWTAs for these applications. GaN-on-SiC based high electron mobility transistors (HEMTs) and monolithic microwave integrated circuits (MMICs) have been demonstrated at microwave and millimeter-wave frequencies with power densities ranging from an order of magnitude at microwave to several times at $\mathrm{mmW}$ higher than those from earlier technologies [3]. GaN technology has showed continuous improvements in output power, efficiency, bandwidth, and reliability with no signs of slowing down [4].

The presence of nonlinearities in transmitter amplifier can have two effects on the overall performance of digital communication systems: (1) waveform distortion; (2) spectral regrowth. Waveform distortion is quantified by error-vector-

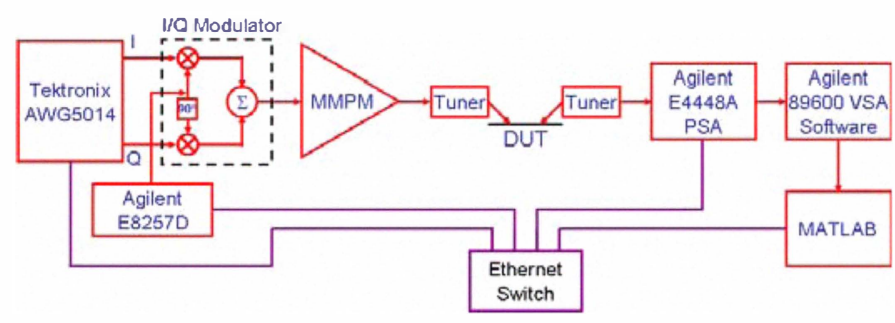

Figure 1. Block diagram of digital waveform system.

magnitude (EVM) which can be related to system level performance metrics such as bit-error-rate (BER). It also limits the use of more spectrally efficient, higher-order modulation schemes. Spectral regrowth is quantified by adjacent-channel-power-ratio (ACPR) which measures power leakage into adjacent channels. This power leakage appears as an effective noise to the adjacent channels and also increases probability of data error. Spectral regrowth limits the number of data channels in a fixed bandwidth. The nonlinear characteristics of GaN HEMT devices and their effects on power amplifier have been investigated at microwave and cellular band frequencies [5]. However, similar studies for $\mathrm{GaN}$ devices and the effects of these nonlinearities on complex digital waveforms at $\mathrm{mmW}$ frequencies remain unexplored.

In this paper, a digital waveform system for characterizing the linearity of GaN HEMT devices and MMICs at $\mathrm{mmW}$ frequencies will be described. To the best of the authors' knowledge, this kind of study at $\mathrm{mmW}$ is the first reported. The paper is organized as follows. In Section II, the description of the digital waveform system is given. In Section III, the design and fabrication of a one-stage Ka-band GaN amplifier is described. In Section IV, the linearity performance of the Ka-band GaN amplifier is characterized using single-tone, two-tone and digitally modulated waveforms. The dependence of its linearity performance on drain bias current is also studied.

\section{DESCRIPTION OF DigitAL WAVEFORM SYSTEM}

The block diagram of the $\mathrm{mmW}$ digital waveform system is shown in Figure 1. It includes a vector signal generator (VSG) and a vector signal analyzer (VSA). The key components of

* Ali Darwish is a visiting scientist at the U.S. Army Research Laboratory from the American University in Cairo, Egypt. 


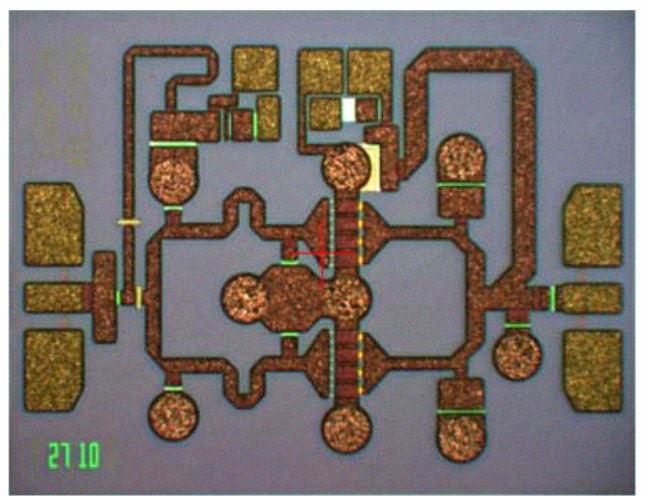

Figure 2. Photo of Ka-band GaN MMIC.

the VSA consist of a Tektronix AWG5014 14-bit, 1.2 GSample/s arbitrary waveform generator (AWG) for generating the digital baseband waveforms, a direct inphase/quadrature (I/Q) modulator for up-converting the baseband waveforms to $\mathrm{mmW}$ carrier frequencies. The local oscillator (LO) for the I/Q modulator is provided by an Agilent E8257D frequency synthesizer with low phase noise option. To provide enough linear drive power, a $30-40 \mathrm{GHz}$ $50 \mathrm{~W}$ millimeter-wave power module (MMPM) is used as the driver amplifier. The MMPM is driven only up to $2 \mathrm{~W}$ output power (at $-14 \mathrm{~dB}$ backoff) to ensure linear operation. This is verified by connecting the digital waveform system in a backto-back configuration. The observed overall gain compression within the entire power range is less than $0.1 \mathrm{~dB}$, and overall phase shift is less than 2 degree. An Agilent E4448A $50 \mathrm{GHz}$ PSA spectrum analyzer with a built-in down-convertor and a 14-bit, 80-MHz-bandwidth digitizer, together with the Agilent 89600 VSA software running on an external computer, is used as the VSA. The Agilent VSA software downloads captured waveforms from the PSA and performs demodulation and analysis on the waveforms.

Both the VSG and the VSA are controlled by a collection of MATLAB programs running on the same computer as the Agilent VSA software. These programs are specifically developed and responsible for generating digital waveforms and downloading them to the AWG, as well as transferring the captured waveforms from the VSA software into the MATLAB environment for further processing. They also perform diagnostics of system imperfections, such as carrier leakage, I/Q imbalance and quadrature error in the $I / Q$ modulator and correct for them. To ensure synchronization, all the instruments in the system share the same clock reference and trigger source. This enables time averaging of multiple waveform traces to help improve signal-to-noiseratio.

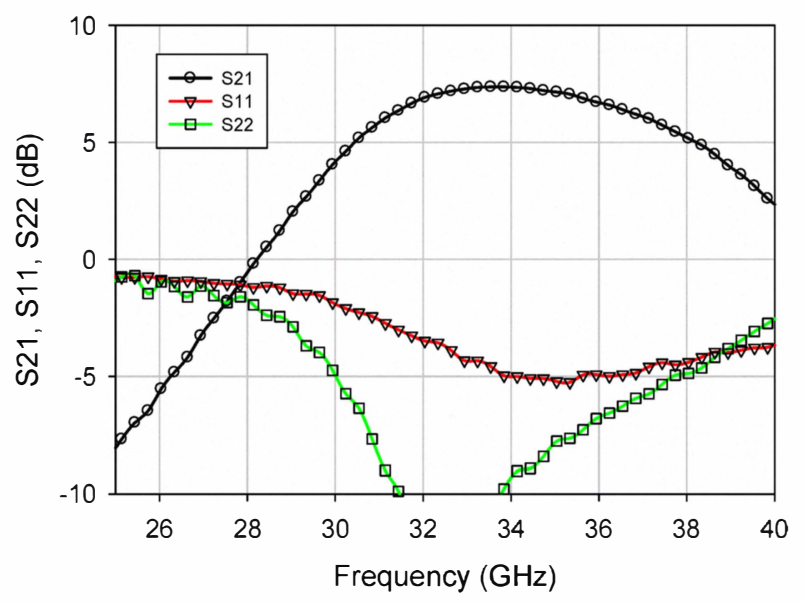

Figure 3. Small signal performance of Ka-band GaN amplifier.

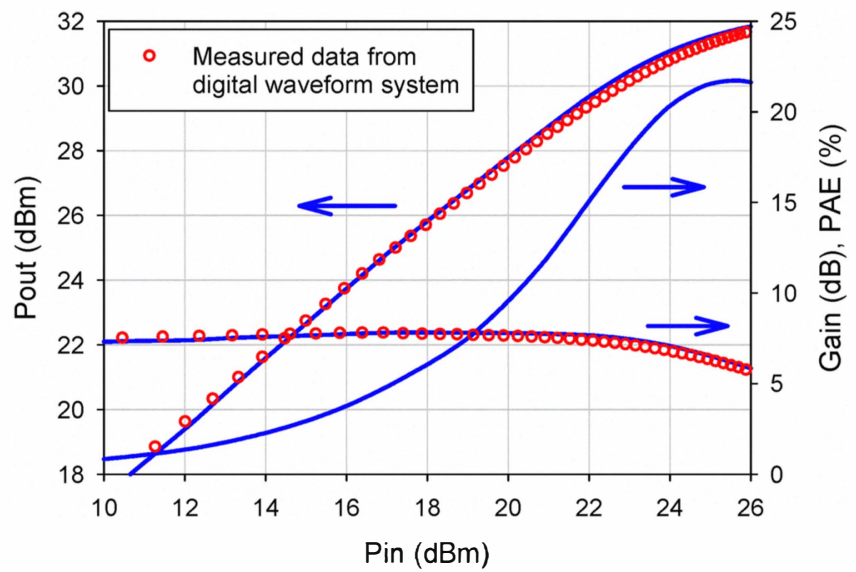

Figure 4. CW Single-tone measurement of Ka-band GaN amplifier.

\section{MMIC DESIGN AND FABRICATION}

A one stage MMIC was fabricated to test the linearity of $\mathrm{GaN} / \mathrm{SiC}$ devices at Ka-band. The MMIC consists of two $8 \times 50 \mu \mathrm{m}$ HEMT cells with $0.25 \mu \mathrm{m}$ gate width. The source drain spacing is $3 \mu \mathrm{m}$, the saturation current (Idss) is 0.8 $\mathrm{A} / \mathrm{mm}$. The AlGaN/GaN HEMT epitaxial layers were grown on 3-inch semi-insulating $6 \mathrm{H}-\mathrm{SiC}$ substrates in an MOCVD reactor. A picture of the MMIC is shown in Figure 2. The MMIC is matched to $50 \mathrm{ohm}$ on the input/output. The input and output matching networks are designed with two poles to increase the bandwidth. The output matching network is matched to the device's power load from $29-36 \mathrm{GHz}$. The input matching network includes a $5 \mathrm{ohm}$ series resistor to ensure unconditional stability and improve the input return loss. The gate and drain biases are accomplished through high impedance quarter wave transformers. Last, DC blocking capacitors are inserted at the input and output to 


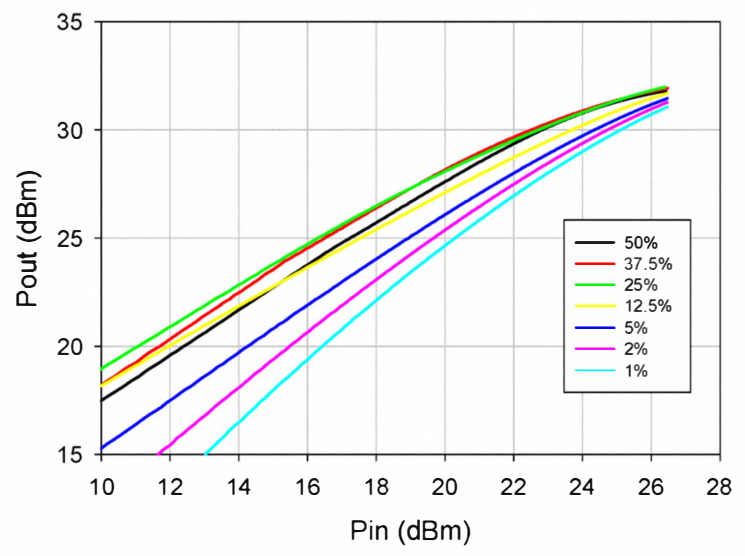

Figure 5. Measured AM/AM transfer curves for different drain

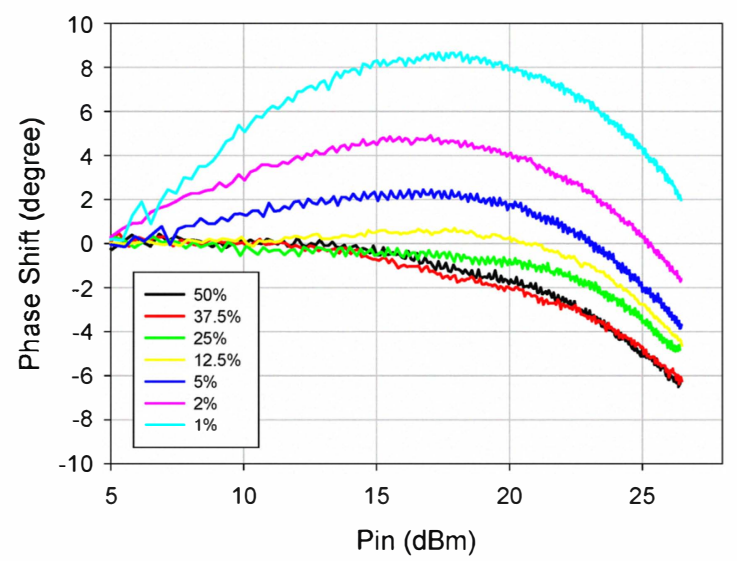

Figure 6. Measured AM/PM transfer curves for different drain current.

eliminate the need for bias tees. The measured small signal performance is shown in Figure 3.

Figure 4 shows the output power, gain, and PAE at 31.5 $\mathrm{GHz}$ under $20 \mathrm{~V}$ drain voltage and drain current at $50 \%$ of Idss. The observed $\mathrm{P} 1 \mathrm{~dB}$ is $30.8 \mathrm{dBm}$, and at peak PAE of $25 \%$, Pout is $31.7 \mathrm{dBm}$. The power measurements were done with conventional thermocouple power sensors and power meters under $\mathrm{CW}$ driven condition. Also shown in Figure 5 is power sweep measurement obtained from a second method using the digital waveform system (which will be described in more detail later). The two techniques show excellent agreement with each other.

\section{DIGITAL WAVEFORM CHARACTERIZATION}

\section{A. Single-tone waveform measurements}

To measure the single-tone responses of the GaN MMIC PA, the VSG was programmed to generate step waveforms with 256 power levels. The power transfer characteristics of the PA were measured by comparing its responses at these different power levels. This technique has been shown to

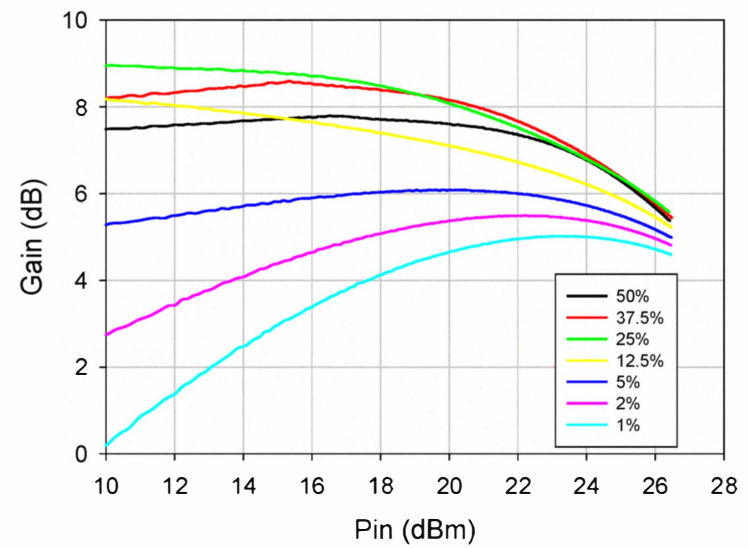

Figure 7. Measured Gain Compression curves for different

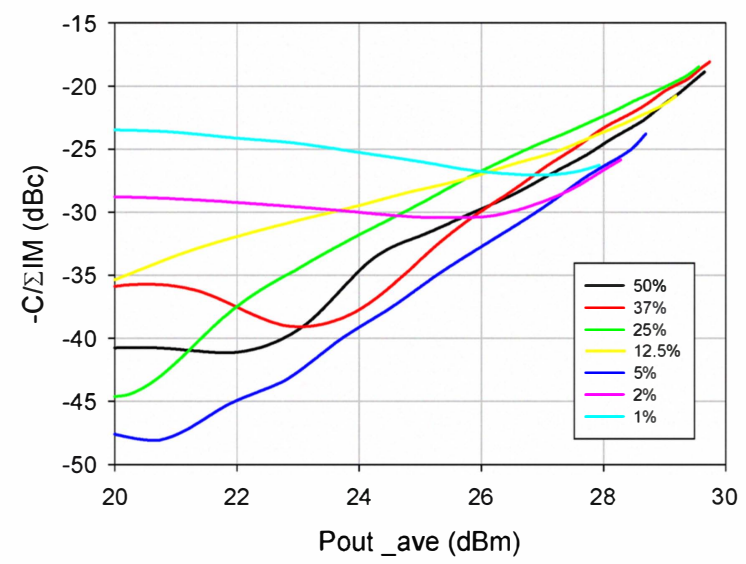

Figure 8. Measured C/SIM.

produce good agreement with those from conventional power scanning technique (Figure 5). Furthermore, because of the vector signal nature of the generator and analyzer, both $\mathrm{AM} / \mathrm{AM}$ and $\mathrm{AM} / \mathrm{PM}$ responses are obtained. In Figures 5, 6 and 7, the measured AM/AM and AM/PM and gain compression curves at $31.5 \mathrm{GHz}$ are plotted for $20 \mathrm{~V}$ drain bias and different quiescent drain current settings expressed in percentage of Idss. In general, the AM/PM effect of this GaN MMIC as observed in Figure 6 is not significant except for very low value of drain current; it is therefore not expected to have major impact on the overall linearity of the PA. The power and gain, however, show significant variation for different drain bias current.

To assess the overall linearity, it is noted that, for class A or similar biasing ( $\sim 50 \%$ Idss), the gain curves show relative "soft" compression characteristics. This is observed by the large separation in input power between maximum linear power and saturation (Figure 7). It is noteworthy that the gain at saturation is not strongly dependent on the bias. This is because, at large input drive, the transistor is almost completely turned on by the RF. On the other hand, at large back off from saturation, the small signal gain depends 


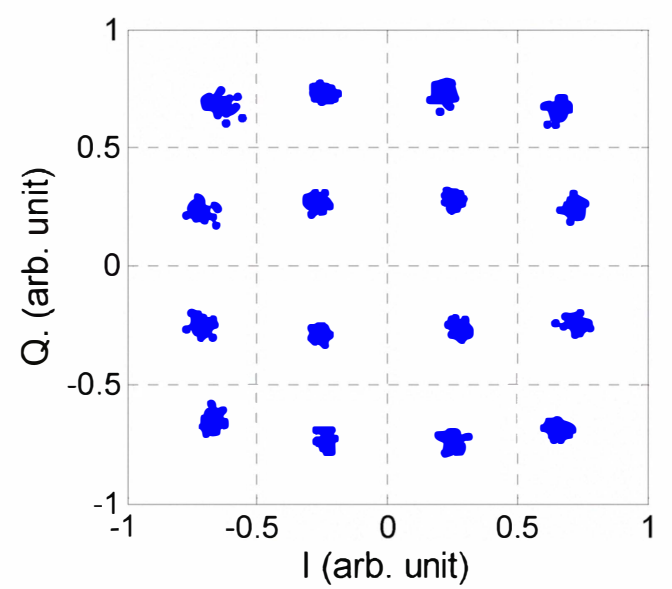

Figure 9. Recovered 16-QAM constellation.

strongly on the bias. Low drain bias current results in lower gain. A balance between small signal gain and saturated gain which results in a flatter gain response is observed for drain current set to $5 \%$ of Idss.

\section{B. Two-tone waveform measurements}

In figure $7, \mathrm{C} / \Sigma \mathrm{IM}$ is plotted as a function of output power at $31.5 \mathrm{GHz}$ for different drain current. $\mathrm{C} / \Sigma \mathrm{IM}$ is defined as the ratio between the total power in the two carriers to the total power in all the intermodulation products $\left(3^{\text {rd }}\right.$ order, $5^{\text {th }}$ order, etc.). The linearity advantage for drain bias current at $5 \%$ of Idss is also observed for two-tone waveform.

\section{Digitally-modulated waveform measurements}

The GaN amplifier was tested with 16-QAM waveform at $31.5 \mathrm{GHz}$. The test waveform had a symbol rate of 10 MSymbols/s, and was filtered by identical matching rootraised-cosine filters with a roll-off factor of 0.4 in both generator and analyzer. The total bandwidth of the input waveform was therefore $14 \mathrm{MHz}$. In Figure 9, an example of a recovered constellation plot showing the effect of nonlinear distortions in the amplifier is shown. The presence of gain compression causes the outer most symbols to derivate from their ideals position, and raises the overall EVM. The corresponding EVM for Figure 9 is about 4.7\%. Spectral regrowth due to nonlinear distortion for the same waveform in Figure 9 is shown in Figure 10. The corresponding ACPR for Figure 10 is about $-28 \mathrm{dBc}$. The center of the adjacent channel is defined to be 1.5 symbol rate $(15 \mathrm{MHz})$ away from the center of the main channel.

\section{CONCLUSION}

A specific, digital waveform system capable of generating and analyzing a wide range of digital waveforms was

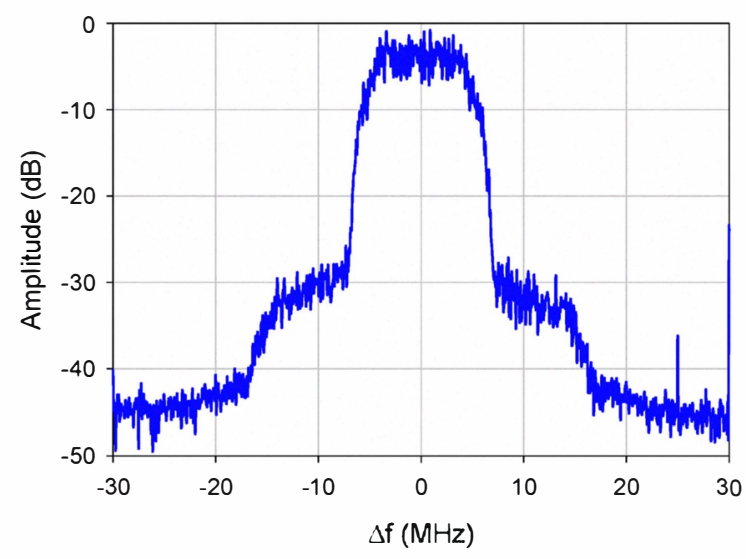

Figure 10. Spectral regrowth due to nonlinear distortion.

developed to study the linearity characteristics of $\mathrm{GaN}$ devices at $\mathrm{mmW}$ frequencies. The linearity of a sample, Ka-band GaN MMIC PA was characterized. The linearity of the PA showed strong dependence on the drain bias current. Optimum linearity was achieved by balancing the overall small signal and saturated gain. This linearity enhancement was observed for single-tone, two-tone waveforms. The system has been proven to provide useful information on linearity characteristics and is currently used in conjunction with a $\mathrm{mmW}$ load-pull system to study the I/O match of GaN devices for optimal power, PAE, and linearity performance.

\section{ACKNOWLEDGEMENT}

The authors would like to thank Dr. T. Ivanov for useful discussions and support.

\section{REFERENCES}

[1] K. Singarajah and D. Bestwick, "Delivering Ka-band Milsatcom Services: Opportunities and Challenges," IET Seminar on Milsatcom, June 2010, London, UK

[2] H. Beljour, L. Coryell, T. Fung, J. Gallagher, R. Hoffmann, G. Michael and J. Shields, "Army SATCOM On The Move Technology Initiatives," MILCOM2009, October, 2009, Boston, MA

[3] A. M. Darwish, K. Boutros, B. Luo, B. D. Huebschman, E. Viveiros, and H. A. Hung, "AlGaN/GaN Ka-band 5-W MMIC Amplifier," IEEE Trans. Microwave Theory \& Tech., vol. 54, no. 12, pp. 4456-4463, December 2006.

[4] M. J. Rosker, J. D. Albrecht, E. Cohen, J. Hodiak and T.-H. Chang, "DARPA's GaN Technology Thrust," IMS2010 Digest, pp. 1214-1217, May, 2010, Anaheim, CA.

[5] P. M. Cabral, J. C. Pedro and N. B. Carvalho, "Nonlinear Device Model of Microwave Power GaN HEMTs for High Power-Amplifier Design," IEEE Trans. Microwave Theory \& Tech., vol. 52, no. 11, pp. 2585-2592, November 2004. 